\title{
Socio-Demographic Inequalities in HIV Testing and Prevalence Among Older Adults in Rural Tanzania, 2013
}

\author{
Angelina C. Mtowa*1, Annette A. Gerritsen², Sally Mtenga', Mary Mwangome ${ }^{1}$ and \\ Eveline Geubbels ${ }^{1}$
}

${ }^{1}$ Impact Evaluation, Ifakara Health Institute, Morogoro, United Republic of Tanzania; ${ }^{2}$ EPI Results, Pietermaritzburg, South Africa

\section{Objective}

This study aimed to estimate socio-demographic inequalities in HIV testing and prevalence among adults aged 50+ years, living in Ifakara town, Tanzania, in 2013

\section{Introduction}

Like in other African countries, most HIV research in Tanzania focuses on adults 15-49 years, ignoring persons aged 50 years and above. In Tanzania, the HIV testing rate (ever tested) for 15 - 49 year olds has increased from $37 \%$ to $62 \%$ for women and $27 \%$ to $47 \%$ for men between 2008 and 2012. Limited data is available on HIV testing and prevalence among older adults specifically. Some studies in Sub-Saharan Africa have, however, reported a high HIV prevalence among older people.

\section{Methods}

This is a cross-sectional study using data from the baseline measurement of the Ifakara MZIMA cohort study conducted in 2012/13. Consenting participants were interviewed and tested for HIV. Information on prior testing behaviour, age, marital status, occupation, gender, ethnicity, religion, education (PROGRESS framework indicators) was retrieved from the interviewer administered questionnaires. Multivariable logistic regression analysis was used to establish associations between HIV testing and prevalence with the socio-demographic indicators.

\section{Results}

Among the 1,643 adults 50+ years included in the study, HIV prevalence and the HIV testing rate (ever tested) were $6 \%$ and $11.4 \%$ respectively. Multivariable analysis showed that the HIV testing rate was lower for older people $(\mathrm{OR}=0.19(95 \% \mathrm{CI} 0.09-0.41$ for $75+$ versus 50-54); those separated/divorced/widowed had higher odds of testing than those married ( $\mathrm{OR}=1.46 ; 1.02-2.10)$; and "other Christians" had a higher odds than Muslims $(\mathrm{OR}=1.95 ; 1.06-3.58)$. With respect to HIV prevalence, it is higher for older people $(\mathrm{OR}=0.27 ; 0.11-0.66$ for 75+ versus 50-54); and Catholics have a lower odds compared to Muslims (OR=0.54; 0.34-0.85).

\section{Conclusions}

These results are valuable as they provide insight into the sociodemographic inequalities among older adults. The high HIV prevalence among this group and the low HIV testing behaviour call for more efforts on HIV prevention, treatment and care. Also the older adults should be included in the national surveillance systems like DHS and THMIS as they are also the HIV risk population. Furthermore, the government should establish elder friendly services and strengthening the capacity of the health system to deliver quality services for HIV and other diseases in the country.Additionally, the findings of this study also warrant further research on the HIV prevalence and testing behaviour of older adults, including studies on the sexual behaviour of older adults

\section{Keywords}

HIV; Older adults; Testing; prevalence; social economic inequalities

\section{Acknowledgments}

This study was supported by health information systems grant from Global Fund round 9; the author would like to acknowledge the support from the Ministry of Health and Social Welfare (MoHSW), Kilombero district, IHI staff, and the local community of Ifakara.

\section{References}

1. Davis T, Zanjani F. Prevention of HIV Among Older Adults: A Literature Review and Recommendations for Future Research. Journal of Aging and Health. 2012;24(8):1399-420.

2. Negin J, Mills EJ, Albone R. Continued neglect of ageing of HIV epidemic at UN meeting. The Lancet. 2011;378(9793):768.

3. Mills J, Rammohan A, Awofeso N. Ageing faster with AIDS in Africa. The Lancet. 2011;377(9772):1131-3.

4. UNAIDS. The Gap report, Beginning of the end of the AIDS epidemic. Geneva: UNAIDS, 2014.

5. UNAIDS. HIV and Aging, A special supplement to the UNAIDS report on the global AIDS epidemic 2013. Geneva, Switzerland, 2013.

6. Negin J, Cumming R. HIV infection in older adults in sub-Saharan Africa: extrapolating prevalence from existing data. Bulletin of the World Health Organization. 2010;88:847-53.

7. Shisana O, et al. South African National HIV Prevalence, Incidence and Behaviour Survey, 2012. Cape Town: HSRC Press;. 2014.

8. Gómez-Olivé F, et al. Prevalence of HIV among those 15 and older in rural South Africa. AIDS CARE. 2013;25(9):1122-8.

9. Negin J, et al. HIV attitudes, awareness and testing among older adults in Africa. AIDS and Behavior. 2012;16(1):63-8

10. WHO. Universal Access Progress Report. Geneva: UNAIDS, 2011.

11. Tanzania Commission for AIDS (TACAIDS) ZACZ, National Bureau of Statistics (NBS), Office of the Chief Government Statistician (OCGS), and ICF International. Tanzania HIV/AIDS and Malaria Indicator Survey 2011-12: Key Findings. Dar Es Salaam, Tanzania: 2012.

12. Hajizadeh M. Socioeconomic inequalities in HIV/AIDS prevalence in sub-Saharan African countries: evidence from the Demographic Health Surveys. International journal for equity in health. 2014;13:1-2.

\footnotetext{
*Angelina C. Mtowa

E-mail: amtowa2001@yahoo.co.uk
} 